\title{
The Application of the Capacitor's Physics to Optimize Capacitive Angular-Position Sensors
}

\author{
Xiujun Li, Gerben de Jong, and Gerard C. M. Meijer, Member, IEEE
}

\begin{abstract}
The distributions of the potential and charge density in a capacitive angular-position sensor with three electrode disks in an axis-symmetrical configuration have been found by creating a suitable physical model and solving Laplace's equation. The influences of geometrical parameters on the nonlinearity, which originates from the electric-field-bending effect, are discussed in detail for a capacitive angular-position sensor. The approximately analytical results are verified by a numerical analysis and are found to be very useful to predict the influences of geometrical nonidealities. The calculations are experimentally verified by using a novel, absolute angular-position sensor with a nonlinearity of less than \pm 17 " and \pm 50 " over measurement ranges of $15^{\circ}$ and $90^{\circ}$, respectively.
\end{abstract}

Index Terms - Capacitance tranducers, geometric modeling, position measurement.

\section{INTRODUCTION}

A LOW ENERGY consumption and a simple construction are some of the attractive features of capacitive sensors. In the past, the accuracy of capacitive sensors was limited due to the problem of measuring the small currents in the capacitive elements in an accurate way. In some recent papers [1]-[3], [6], it has been shown that a rather high degree of accuracy can be obtained with capacitive sensor elements by applying new concepts. However, the electric-field-bending effect is still one of the major reasons for the remaining inaccuracy of capacitive sensors even though well-designed guard electrodes are used.

In this paper the influences of geometrical parameters on the nonlinearity, due to the electric-field-bending effect, are discussed in detail by analyzing the physical properties of the capacitive elements. It is shown that it is possible to predict the influences of geometrical nonidealities and to optimize the geometrical parameters of the capacitive sensor.

For a proper analysis of the relation between the capacitances and the geometrical parameters, a physical model and mathematical tools are required. Therefore, the analysis in this paper starts with a physical model of the capacitive angularposition sensor. Next, a complete description of potentials, charge densities, and capacitances for a complex symmetrical electrostatic system is obtained by solving Laplace's equation. The analytical results have been experimentally verified using a novel, accurate capacitive angular-position sensor.

Manuscript received August 18, 1995; revised January 17, 1996.

The authors are with the Department of Electrical Engineering, Delft University of Technology, Delft 2628, The Netherlands.

Publisher Item Identifier S 0018-9456(97)00916-9.

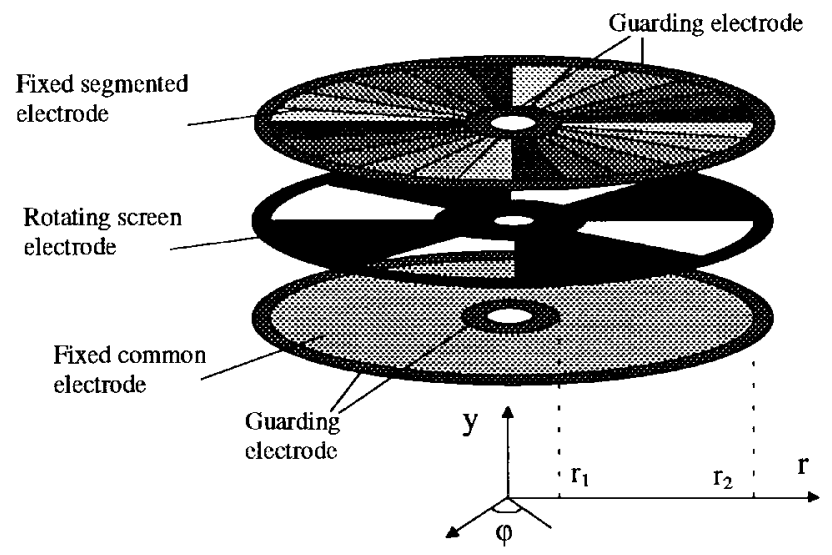

Fig. 1. A simplified structure of a capacitive angular-position sensor with three disk electrodes.

\section{Physical Model of the Capacitive Sensing Element}

\section{A. The Structure and Principle of the Capacitive Sensor}

A novel capacitive sensing element, which consists of three parallel disks, has been presented in [3], [7] (see Fig. 1).

By the use of a cross-quad configuration in the sensing element [3], [7], six capacitances between the segmented electrode and the common electrode are required to be measured. By measuring these capacitances and adapting the approach that uses the ratio of two linear combinations of the capacitances (RTLCC), which has been described in [3] and refers to (10), the angular position of the sensing elements can be determined over the measurement range of $90^{\circ}$. Many undesired mechanical and electronic influences, for instance, the eccentricities, the nonflatness, the obliqueness of the electrodes, and the gain errors and offsets of the signal-processing system, are eliminated or strongly reduced by the uses of the cross-quad configuration, the guarding electrode, and the RTLCC approach [3], [7]. However, a nonlinearity due to the electric-field-bending effect still remains.

\section{B. Physical Model of the Sensor}

Generally, it is difficult to find an analytic solution for a three-dimensional electrostatic-field problem. However, when the potential distribution in the geometry depends only on twodimensional coordinates and when there is a homogeneous permittivity inside, an analytical solution can usually be found [4], [5]. 


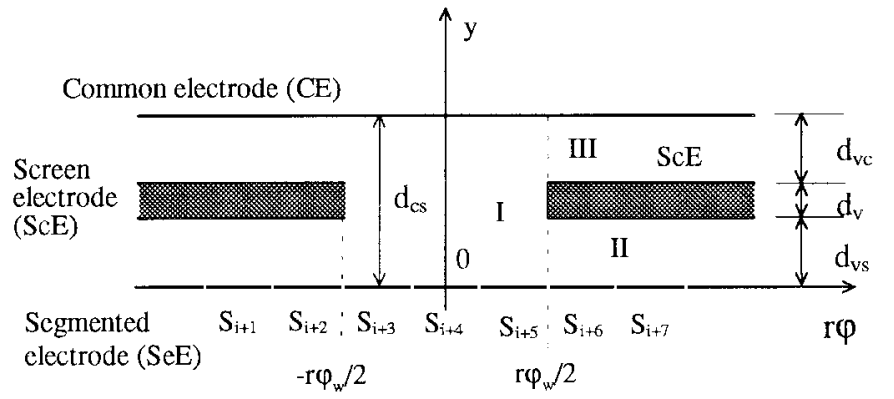

Fig. 2. A crossview of a part of the sensor.

Fortunately, the electrode configuration which is shown in Fig. 1 can be simplified to a two-dimensional structure, and the electrostatic-field problem in polar coordinates is transformed to one in rectangular coordinates.

If the dimensions of the segmented and common electrodes in the radial direction are much larger than the electrode distances and larger than the dimensions of the electrodes in the tangential direction, we can simplify the electrostaticfield problem by supposing that the potential is not directly affected by the radial dimension $r$ at all. The effect of the radial dimension $r$ on the potential is indirectly taken into account via the tangential dimension [3]. A two-dimensional model in rectangular coordinates, corresponding to the threedimensional structure shown in Fig. 1, is shown in Fig. 2, which is symmetrical with respect to the $y$-axis.

In Fig. 2, $\varphi_{\mathrm{w}}$ is the angular width of the screen windows, $d_{\mathrm{cs}}$ the distance between the common and the segmented electrodes, $d_{\mathrm{v}}$ the thickness of the screen electrode, $d_{\mathrm{vs}}$ the distance between the screen and the segmented electrodes, $d_{\mathrm{vc}}$ the distance between the common and the screen electrodes. Now, the parameter $r$ is supposed to be constant. Then $r \varphi$ is the coordinate in the tangent direction at a radial distance $r$. Thus only the two-dimensional Laplace's equation has to be solved in rectangular coordinates.

In order to calculate the capacitance between the common electrode (CE) and the segmented electrode (SeE), we choose the common electrode as the transmitting electrode with potential $U_{0}$ and the segmented electrode as the receiving electrode with zero potential (i.e., grounded). The effect of the gap between two adjacent segments of the segmented electrode is neglected. The screen electrode ( $\mathrm{ScE}$ ) is also connected to ground.

For the total solution of this two-dimensional electrostaticfield problem, the configuration is divided into three areas I, II, and III (see Fig. 2) with generally different boundary conditions. For both $\varphi=\varphi_{\mathrm{w}} / 2$ and $\varphi=-\varphi_{\mathrm{w}} / 2$ within the range $0<y<d_{\mathrm{cs}}$, we assume that the potential is a continuous piecewise-linear potential, taken as a basic triangular-shaped function. The accuracy of this approximation is verified by the numerical calculation described in Section IV. This potential is shown in Fig. 3.

In this model, the potential $U_{0}$ represents the potential of the transmitting electrode. The potentials $U_{1}, U_{2}$ and positions $d_{1}, d_{2}$ are auxiliary variables which are determined by the potential $U_{0}$, the geometrical parameters $\left(d_{\mathrm{cs}}, d_{\mathrm{v}}, d_{\mathrm{vs}}\right.$

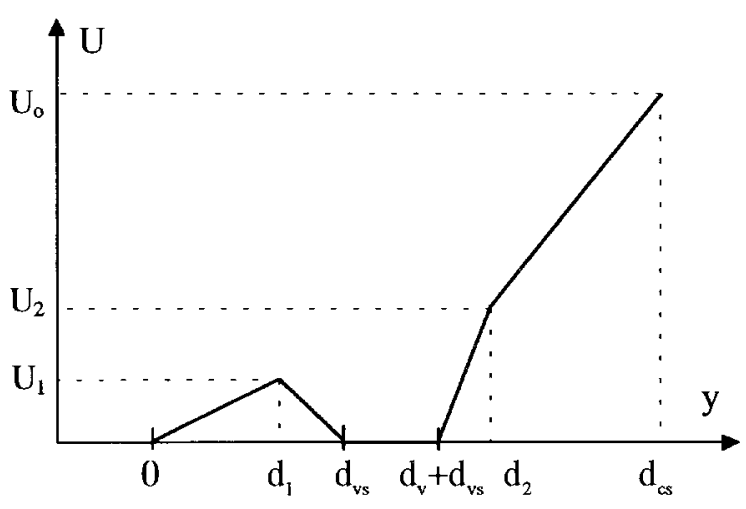

Fig. 3. The piecewise linear approximation for the potential at $\varphi=\varphi_{\mathrm{w}} / 2$.

and $d_{\mathrm{vc}}$ ) and the width of the screen windows $\varphi_{\mathrm{w}}$. In the following section we give the solution of the two-dimensional electrostatic-field problem and discuss the relations between $U_{1}, U_{2}, d_{1}, d_{2}$ and the geometrical parameters.

\section{The Solution of the Two-Dimensional Electrostatic-Field Problem}

By solving Laplace's equation in the areas I, II, and III and according to Gauss's flux theorem [4], for the distribution of the surface charge density $\sigma_{\mathrm{r}}\left[\mathrm{C} / \mathrm{m}^{2}\right]$ on the surface of the receiving electrode $(\mathrm{SeE})(y=0)$ it can be found that

$$
\sigma_{r}=\left\{\begin{array}{c}
-\varepsilon \frac{U_{0}}{d_{\mathrm{cs}}}+\varepsilon U_{0} \sum_{n=1}^{\infty} \frac{n \pi}{d_{\mathrm{cs}}} f_{1}(n) \frac{\cosh \left(\lambda_{1} r \varphi\right)}{\cosh \left(\lambda_{1} r \varphi_{\mathrm{w}} / 2\right)}, \\
-\frac{\varphi_{\mathrm{w}}}{2}<\varphi<\frac{\varphi_{\mathrm{w}}}{2} \\
-\varepsilon U_{0} \sum_{n=1}^{\infty} \frac{n \pi}{d_{\mathrm{vs}}} f_{2}(n) e^{-\lambda_{2} r\left(\varphi-\varphi_{w} / 2\right)}, \frac{\varphi_{\mathrm{w}}}{2}<\varphi
\end{array}\right.
$$

where

$$
\begin{gathered}
f_{1}(n)=\frac{2 d_{\mathrm{cs}}}{(n \pi)^{2}}\left\{\frac{\sin \left(\lambda_{1} d_{2}\right)}{d_{\mathrm{cs}}-d_{2}}+\frac{U_{1}}{U_{0}}\left[\frac{\sin \left(\lambda_{1} d_{\mathrm{vs}}\right)}{d_{\mathrm{vs}}-d_{1}}-\frac{d_{\mathrm{vs}} \sin \left(\lambda_{1} d_{1}\right)}{\left(d_{\mathrm{vs}}-d_{1}\right) d_{1}}\right]\right. \\
+\frac{U_{2}}{U_{0}}\left[\frac{\sin \lambda_{1}\left(d_{\mathrm{vs}}+d_{\mathrm{v}}\right)}{\left(d_{2}-d_{\mathrm{vs}}-d_{\mathrm{v}}\right)}\right. \\
\left.\left.-\frac{d_{\mathrm{vc}} \sin \left(\lambda_{1} d_{2}\right)}{\left(d_{2}-d_{\mathrm{vs}}-d_{\mathrm{v}}\right)\left(d_{\mathrm{cs}}-d_{2}\right)}\right]\right\} \\
f_{2}(n)=\frac{2 U_{1} d_{\mathrm{vs}}^{2} \sin \left(\lambda_{2} d_{1}\right)}{U_{0}(n \pi)^{2} d_{1}\left(d_{\mathrm{vs}}-d_{1}\right)} \\
\lambda_{1}=n \pi / d_{\mathrm{cs}}, \quad \lambda_{2}=n \pi / d_{\mathrm{vs}}
\end{gathered}
$$

while $n$ is an integer. The variables $U_{1}, U_{2}, d_{1}$ and $d_{2}$ can be found from the common boundary conditions at boundary $\varphi=\varphi_{\mathrm{w}} / 2$ or $\varphi=-\varphi_{\mathrm{w}} / 2$.

If two $y$ values in the range $0<y<d_{\mathrm{vs}}$ and two $y$ values in the range $d_{\mathrm{vs}}+d_{\mathrm{v}}<y<d_{\mathrm{cs}}$ are arbitrarily selected, then four equations with the variables $U_{1}, U_{2}, d_{1}$ and $d_{2}$ are obtained. The relations between the variables $U_{1}, U_{2}, d_{1}$, and $d_{2}$ and the geometrical parameters can be found by solving these four equations. In order to make the theoretical results optimally coincide with the practical results, we select four $y$ values that are respectively the points between $d_{1}$ and $0, d_{1}$ and $d_{\mathrm{vs}}$, 
$d_{\mathrm{vs}}+d_{\mathrm{v}}$ and $d_{2}$, and $d_{2}$ and $d_{\mathrm{cs}}$ namely

$$
\begin{aligned}
& y=2 d_{1} / 3, y=\left(2 d_{1}+d_{\mathrm{vs}}\right) / 3, y=\left(2 d_{2}+d_{\mathrm{vs}}+d_{\mathrm{v}}\right) / 3 \\
& y=\left(2 d_{2}+d_{\mathrm{cs}}\right) / 3
\end{aligned}
$$

According to the definition of a capacitance, the capacitances between the segments on the segmented electrode and common electrode can be obtained from (1). The capacitances $C_{i+1} \cdots C_{i+6}$ respectively corresponding to the segments $\mathrm{S}_{\mathbf{i}+1} \cdots \mathrm{S}_{\mathbf{i}+6}$ (see Fig. 2) are

$$
\begin{aligned}
C_{i+4}-C_{i+1} & =\varepsilon \frac{\varphi_{\mathrm{s}}\left(r_{2}^{2}-r_{1}^{2}\right)}{2 d_{\mathrm{cs}}}\left(1+\frac{\delta_{\mathrm{s}}\left(\varphi_{\mathrm{p}}\right)}{\varphi_{\mathrm{s}}}\right) \\
\left(C_{i+5}+C_{i+6}\right) & -\left(C_{i+2}+C_{i+3}\right) \\
& =\varepsilon \frac{\varphi_{\mathrm{p}}\left(r_{2}^{2}-r_{1}^{2}\right)}{d_{\mathrm{cs}}}\left(1+\frac{\delta_{\mathrm{p}}\left(\varphi_{\mathrm{p}}\right)}{\varphi_{p}}\right) \\
C_{i+5}-C_{i+2} & =\varepsilon \frac{\varphi_{\mathrm{s}}\left(r_{2}^{2}-r_{1}^{2}\right)}{2 d_{\mathrm{cs}}}\left(1+\frac{\delta_{25}\left(\varphi_{\mathrm{p}}\right)}{\varphi_{\mathrm{s}}}\right)
\end{aligned}
$$

where $\varphi_{\mathrm{p}}$ is the angular position to be measured, $\varphi_{\mathrm{S}}$ is the angular width of an electrode segment $\left(\varphi_{\mathrm{s}}=15^{\circ}\right)$, the parameters $r_{1}$ and $r_{2}$ are the radial dimensions of the sensor (see Fig. 1$), \delta_{\mathrm{p}}\left(\varphi_{\mathrm{p}}\right), \delta_{\mathrm{s}}\left(\varphi_{\mathrm{p}}\right)$ and $\delta_{25}\left(\varphi_{\mathrm{p}}\right)$ are nonlinear terms caused by the electric-field-bending effect, it is holds that (see (7)-(9) at bottom of page).

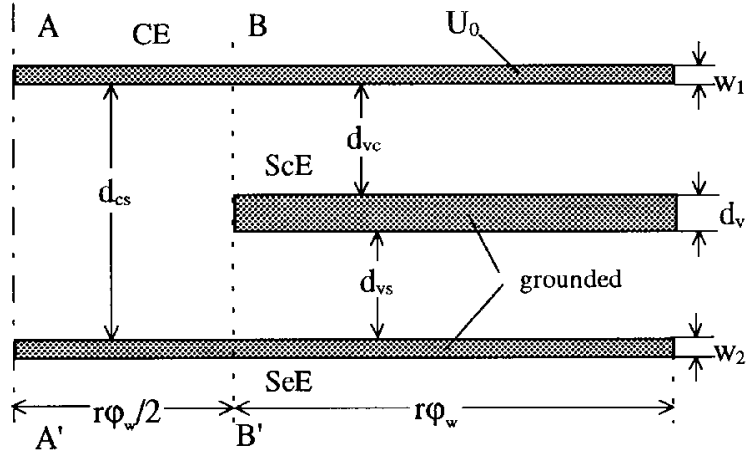

Fig. 4. The model used for a numerical verification of the analysis.

\section{INFLUENCE OF THE ELECTRIC-FIELD-BENDING EFFECT ON THE ACCURACY}

For the sensor structure given in Fig. 1, the relation of the measured angular position $\varphi_{m}$ and the capacitances is represented by (10) [3], [7] (see bottom of page) for $-1 / 2 \leq$ $\left(\varphi_{m} / \varphi_{\mathrm{s}}-i\right) \leq 1 / 2$ where $\alpha$ is called the fine-tuning factor $(0 \leq \alpha \ll 1)$. The appropriate fine-tuning factor will reduce the influence of the electric-field-bending effect.

Because of the cross-quad connection of the segments on the segmented electrode and the symmetrical structure of the rotating screen electrode with four identical windows in Fig. 1

$$
C_{\mathrm{i}+6}=C_{\mathrm{i}} \text {. }
$$

Substituting the capacitance's functions that are described by (6) into (10), results in an equation of the form

$$
\varphi_{m}=\frac{\varphi_{\mathrm{p}}+\delta_{\mathrm{p}}\left(\varphi_{\mathrm{p}}\right)}{1+\delta_{\mathrm{s}}\left(\varphi_{\mathrm{p}}\right) / \varphi_{\mathrm{s}}+\delta_{\mathrm{c}}\left(\varphi_{\mathrm{p}}\right) / \varphi_{\mathrm{s}}}
$$

$$
\begin{gathered}
\delta_{\mathrm{s}}\left(\varphi_{\mathrm{p}}\right)=-\frac{2 d_{\mathrm{cs}}}{r_{2}^{2}-r_{1}^{2}}\left[2 \sum_{n=1}^{\infty} f_{1}(n) \cdot \int_{r_{1}}^{r_{2}} \frac{\cosh \left(\lambda_{1} r \varphi_{\mathrm{p}}\right) \sinh \left(\lambda_{1} r \varphi_{\mathrm{s}} / 2\right)}{\cosh \left(\lambda_{1} r \varphi_{\mathrm{w}} / 2\right)} d r+\sum_{n=1}^{\infty} f_{2}(n) \int_{r_{1}}^{r_{2}} e^{-\lambda_{2} r\left(\frac{5}{2} \varphi_{\mathrm{s}}-\frac{1}{2} \varphi_{\mathrm{w}}-\varphi_{\mathrm{p}}\right)}\left(1-e^{-\lambda_{2} r \varphi_{\mathrm{s}}}\right) d r\right] \\
\delta_{\mathrm{p}}\left(\varphi_{\mathrm{p}}\right)=-\frac{d_{\mathrm{cs}}}{r_{2}^{2}-r_{1}^{2}}\left[2 \sum_{n=1}^{\infty} f_{1}(n) \cdot \int_{r_{1}}^{r_{2}} \frac{\cosh \left(\lambda_{1} r \varphi_{\mathrm{s}} / 2\right) \sinh \left(\lambda_{1} r \varphi_{\mathrm{p}}\right)}{\cosh \left(\lambda_{1} r \varphi_{\mathrm{w}} / 2\right)} d r+2 \sum_{n=1}^{\infty} f_{2}(n) \int_{r_{1}}^{r_{2}} e^{-\lambda_{2} r\left(\frac{5}{2} \varphi_{\mathrm{s}}-\frac{1}{2} \varphi_{\mathrm{w}}\right)} \sinh \left(\lambda_{2} r \varphi_{\mathrm{p}}\right) d r\right] \\
\delta_{25}\left(\varphi_{\mathrm{p}}\right)=-\frac{2 d_{\mathrm{cs}}}{r_{2}^{2}-r_{1}^{2}}\left[2 \sum_{n=1}^{\infty} f_{1}(n) \cdot \int_{r_{1}}^{r_{2}} \frac{\cosh \left(\lambda_{1} r\left(\varphi_{\mathrm{s}}-\varphi_{\mathrm{p}}\right)\right) \sinh \left(\lambda_{1} r \varphi_{\mathrm{s}} / 2\right)}{\cosh \left(\lambda_{1} r \varphi_{\mathrm{w}} / 2\right)} d r\right. \\
\left.+\sum_{n=1}^{\infty} f_{2}(n) \int_{r_{1}}^{r_{2}} e^{-\lambda_{2} r\left(\frac{3}{2} \varphi_{\mathrm{s}}-\frac{1}{2} \varphi_{\mathrm{w}}+\varphi_{\mathrm{p}}\right)}\left(1-e^{-\lambda_{2} r \varphi_{\mathrm{s}}}\right) d r\right]
\end{gathered}
$$

$$
\frac{\varphi_{m}}{\varphi_{\mathrm{s}}}=i+\frac{\left(C_{\mathrm{i}+5}+C_{\mathrm{i}+6}\right)-\left(C_{\mathrm{i}+2}+C_{\mathrm{i}+3}\right)}{2\left(C_{\mathrm{i}+4}-C_{\mathrm{i}+1}\right)+\alpha\left(C_{\mathrm{i}+2}+C_{\mathrm{i}+4}+C_{\mathrm{i}+6}-C_{\mathrm{i}+1}-C_{\mathrm{i}+3}-C_{\mathrm{i}+5}\right)}, \quad i=(0,1, \ldots, 5)
$$




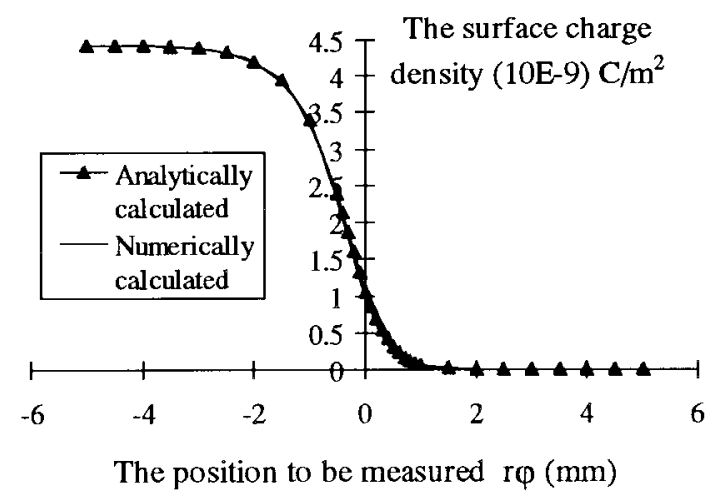

(a)

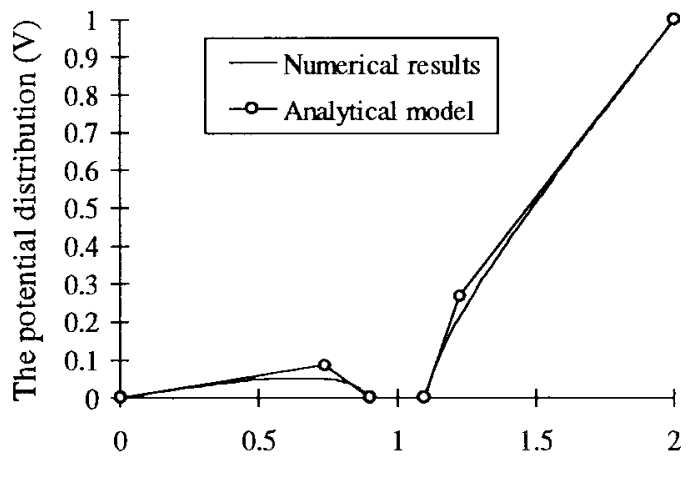

The position on the line B-B' (mm)

(b)

Fig. 5. The distributions of the charge-density and potential analytically and numerically.

where $\delta_{\mathbf{c}}\left(\varphi_{\mathrm{p}}\right)$ is the fine-tuning compensation term for the electric-field-bending effect and

$$
\delta_{\mathrm{c}}\left(\varphi_{\mathrm{p}}\right) \cong \alpha\left(\varphi_{\mathrm{p}}-\frac{\varphi_{\mathrm{s}}}{2}\right) .
$$

With (12), it is found that the systematic nonlinearity $\varepsilon_{e}=$ $\varphi_{m}-\varphi_{\mathrm{p}}$ caused by the electric-field-bending effect can be approximated by

$$
\varepsilon_{e} \cong \delta_{\mathrm{p}}\left(\varphi_{\mathrm{p}}\right)-\frac{\varphi_{\mathrm{p}}}{\varphi_{\mathrm{s}}} \delta_{\mathrm{s}}\left(\varphi_{\mathrm{p}}\right)-\frac{\varphi_{\mathrm{p}}}{\varphi_{\mathrm{s}}} \delta_{\mathrm{c}}\left(\varphi_{\mathrm{p}}\right) .
$$

This nonlinearity is a function of the position $\varphi_{p}$ to be measured, the geometrical parameters $\left(d_{\mathrm{vs}}, d_{\mathrm{v}}, d_{\mathrm{vc}}, d_{\mathrm{cs}}, r_{1}, r_{2}, \varphi_{\mathrm{w}}, \varphi_{\mathrm{s}}\right)$ and the fine-tuning factor $\alpha$.

\section{Numerical SOlution OF THE PROBlem}

In order to verify the validity of the analytical solutions, the distribution of the charge density on the surface of the receiving electrode (SeE) and the potential at $\varphi=\varphi_{\mathrm{w}} / 2$ (see Fig. 2) have been numerically calculated by using an existing Maxwell software package [8]. Fig. 4 shows a scheme of a part of a cross-section view of the sensor which has a symmetrical configuration about the line $\mathrm{A}-\mathrm{A}^{\prime}$. The dimensional and conditional parameters of this configuration are: $w_{1}=w_{2}=0.1 \mathrm{~mm}, d_{\mathrm{v}}=0.2 \mathrm{~mm}, d_{\mathrm{vs}}=d_{\mathrm{vc}}=0.9 \mathrm{~mm}$, $d_{\mathrm{cs}}=2 \mathrm{~mm}, r \varphi_{\mathrm{w}}=15 \mathrm{~mm}$, a potential on the common electrode $U_{0}=1 \mathrm{~V}$, and the screen electrode and segmented electrode are grounded.

In the calculation, about 3000 triangle meshes were used. Fig. 5 shows the numerically and analytically calculated distributions of the charge density on the surface of the receiving electrode ( $\mathrm{SeE}$ ) and of the potentials along the line $\mathrm{B}-\mathrm{B}^{\prime}$ in Fig. 4. The analytical results for the distributions of the charge density are described by (1). From Fig. 5, it can be concluded that analytical and numerical results for distributions of the charge density and potential are in good agreement. The numerical analysis is used mainly to verify the validity of the approximations in the analytical one. However, to predict the influence of small nonidealities, the analytical analysis is much more suited than the numerical one because this kind of
TABLE I

The Geometrical Parameters of the Sensor

\begin{tabular}{|l|c|c|c|}
\hline \multicolumn{1}{|c|}{ parameters } & notation & unit & values \\
\hline electrode distance & $\mathrm{d}_{\mathrm{cs}}$ & $\mathrm{mm}$ & 3.0 \\
thickness of the screen electrode & $\mathrm{d}_{\mathrm{v}}$ & $\mathrm{mm}$ & 0.3 \\
position of the screen electrode & $\mathrm{d}_{\mathrm{v}} \mathrm{d}_{\mathrm{vs}}$ & - & 1.0 \\
inner radix of the electrode & $\mathrm{r}_{1}$ & $\mathrm{~mm}$ & 10.0 \\
outer radix of the electrode & $\mathrm{r}_{2}$ & $\mathrm{~mm}$ & 21.5 \\
width of the segment electrode & $\varphi_{\mathrm{s}}$ & degree & 15 \\
width of the screen electrode & $\varphi_{\mathrm{p}}$ & degree & 45 \\
\hline
\end{tabular}

analysis consumes much less calculation time for the same level of accuracy. These nonidealities are discussed in the following section.

\section{INFLUENCE OF THE GEOMETRICAL PARAMETERS ON THE NONLINEARITY OF THE SENSOR}

The influence of the geometrical parameters $d_{\mathrm{cs}}, d_{\mathrm{vs}}, d_{\mathrm{vc}}$, $d_{\mathrm{v}}, r_{1}, r_{2}, \varphi_{\mathrm{s}}$ and $\varphi_{\mathrm{w}}$ on the nonlinearity of the sensor can be found using (14). Because the relation of the nonlinearity and the geometrical parameters, is very complex, it is difficult to calculate the relation of the nonlinearity and the geometrical parameters in a direct way. The variables $U_{1}, U_{2}, d_{1}$ and $d_{2}$ are also a function of the geometrical parameters and there is an optimum fine-tuning factor $\alpha_{\mathrm{op}}$, which minimizes the influence of the electric-field bending, for every sensor structure. Therefore the variables $U_{1}, U_{2}, d_{1}$, and $d_{2}$ have been calculated, first, then the optimum fine-tuning factor $(\alpha)$ and the corresponding nonlinearity have been calculated, using a trial-and-error method.

Fig. 6 shows the nonlinearity caused by the electric-fieldbending effect over a single-segment range for $\alpha=0$ and $\alpha=0.051$ (optimum value), respectively, for the geometrical parameters listed in Table I.

The nonlinearity is a periodic function with a period of the width of one segmented electrode. By using a fine-tuning factor $\alpha>0$, the nonlinearity of the sensor caused by the electric-field bending effect, can be improved by a factor of 6.5 . 


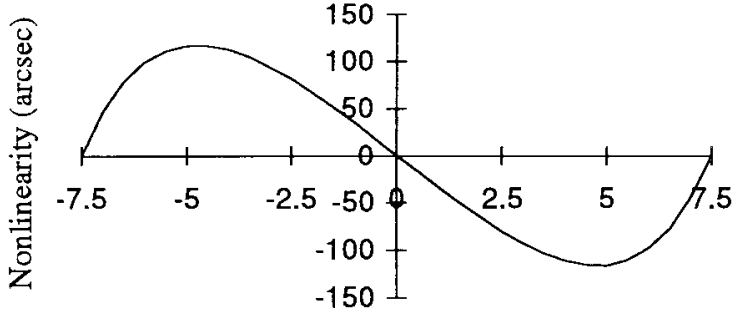

The angular position to be measured for one segment (degree)

(a)

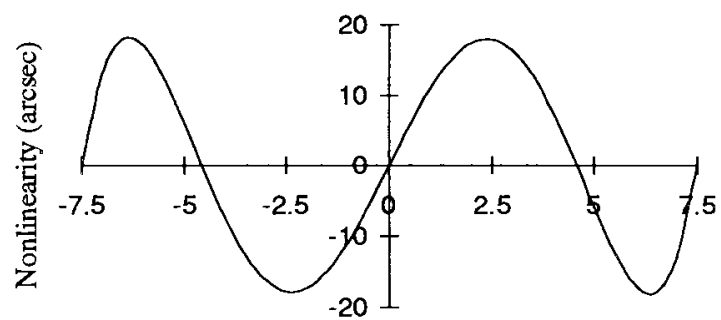

The angular position to be measured for one segment (degree)

(b)

Fig. 6. The nonlinearity of the sensor for (a) $\alpha=0$ and (b) $\alpha=0.051$.

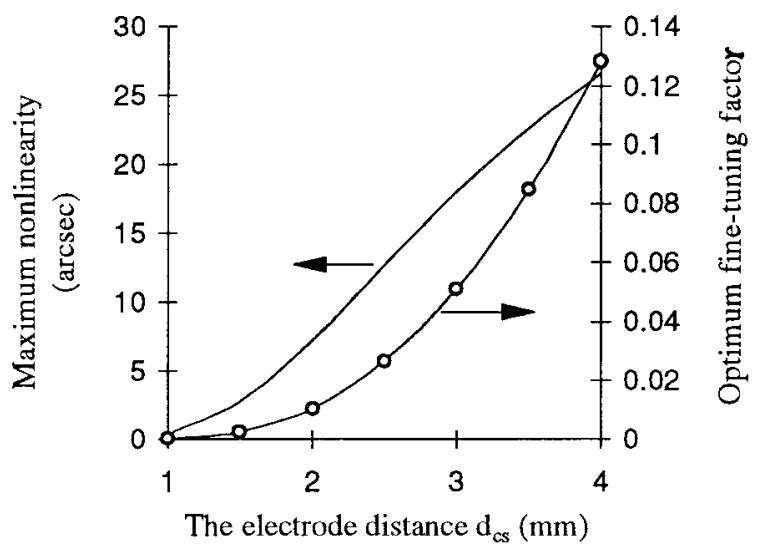

(a)

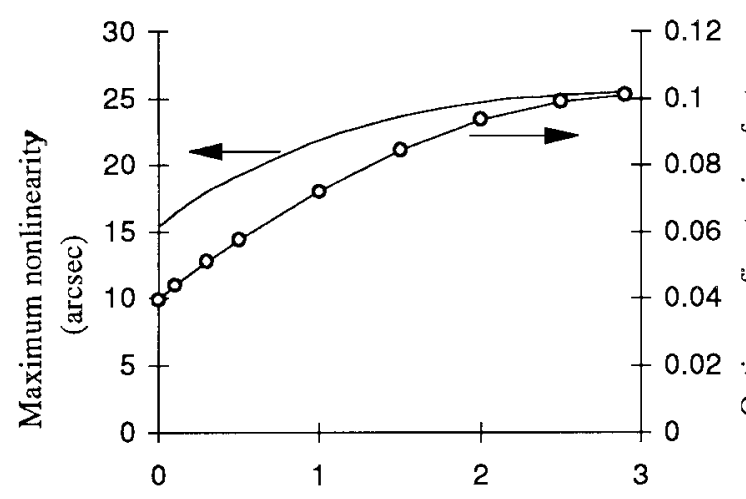

The thickness of the screen electrode $\mathrm{d}_{\mathrm{v}}(\mathrm{mm})$

(b)

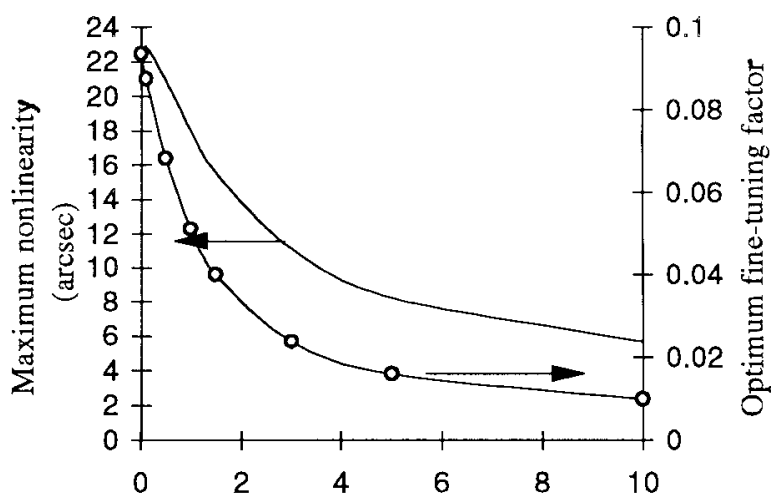

The position of the screen electrode $\mathrm{d}_{\mathrm{v}} / \mathrm{d}_{\mathrm{vs}}$

(c)

Fig. 7. The influence of the electric-field bending on the nonlinearity as function of the geometrical parameters. The electrode structure itself is assumed to be ideal.

Fig. 7 shows the maximum nonlinearity as a function of the vertical geometrical parameters of the sensor $\left(d_{\mathrm{cs}}, d_{\mathrm{v}}\right.$ and $\left.d_{\mathrm{vc}} / d_{\mathrm{vs}}\right)$ and corresponding optimum fine-tuning factor, respectively. In this figure, the maximum nonlinearity is the absolute value of the peak nonlinearity in the range $-\varphi_{\mathrm{s}} / 2 \rightarrow \varphi_{\mathrm{s}} / 2$ [see (Fig. 6(b)].

It is shown that a small nonlinearity is obtained with small electrode distances, a small thickness of the screen electrode, and a large ratio $d_{\mathrm{vc}} / d_{\mathrm{vs}}$ (the screen electrode is near to the segmented electrode). It should be noted here that for accurate sensors a small nonlinearity is not the only requirement. Among the other requirements are a low sensitivity of the angular-position measurement to mechanical tolerances and nonidealities, such as, mechanical vibration, nonflatness, obliqueness and eccentricity of the electrodes, a low temperature coefficient and insensitivity to pollution and humidity. These requirements can be fulfilled better with larger electrode distances and thicknesses, and a smaller ratio $d_{\mathrm{vc}} / d_{\mathrm{vs}}$. The optimum structure is found as a compromise between these conflicting requirements. Fig. 8 shows the re- 


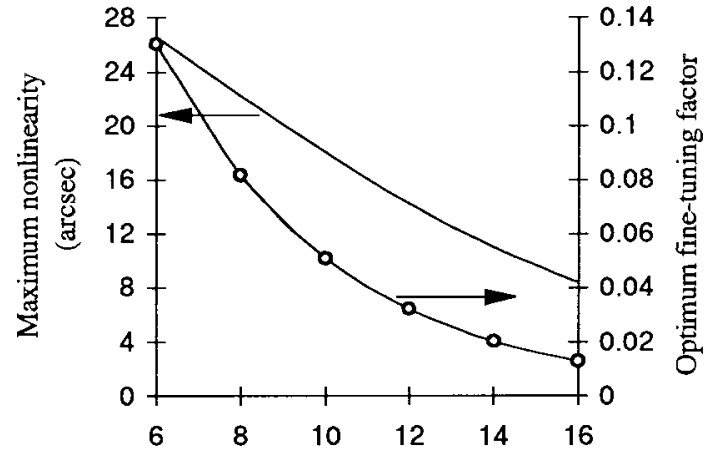

The inner radius $r_{1}$ of the electrodes ( $\left.\mathrm{mm}\right)$

(a)

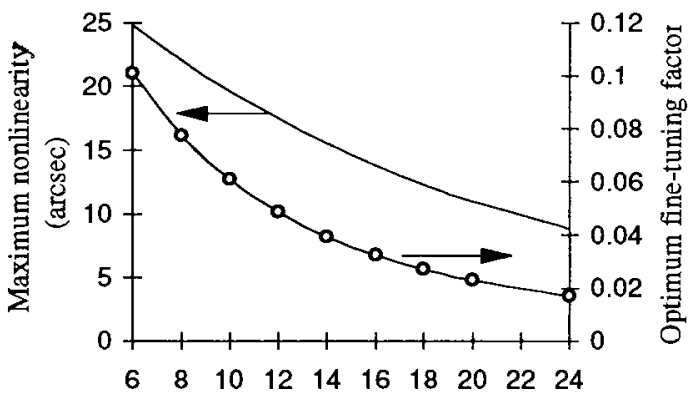

The size of the electrodes in the radial direction $\left(r_{2}-r_{l}\right)(m m)$

(b)

Fig. 8. The influence of the radii of the electrodes on the nonlinearity. (a) $r_{2}-r_{1}$ is constant, (b) $r_{1}$ is constant.

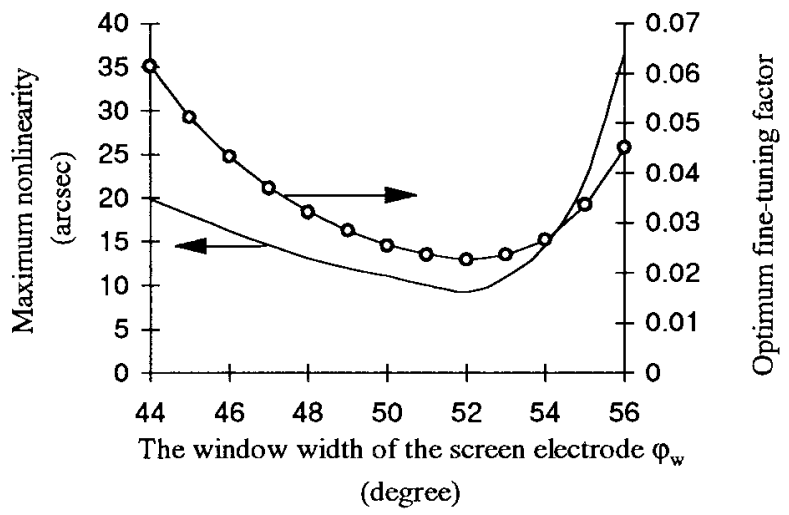

Fig. 9. The optimum window width of the screen electrode.

lations of the maximum nonlinearity and the dimensions of electrodes in the radial direction $\left(r_{1}, r_{2}-r_{1}\right)$ and the corresponding optimum fine-tuning factor.

The larger the dimensions of the electrodes in the radial direction, the smaller is the nonlinearity that is found. This effect is because of the larger width $\left(r \varphi_{\mathrm{S}}\right)$ of the segments. The influence of the electric-field-bending effect in cylindrical coordinates is equivalent to that in rectangular coordinates with a segment width of $x_{\mathrm{s}}=\left(r_{2}+r_{1}\right) \varphi_{\mathrm{s}} / 2$ being the average width of one cylindrical segment. Both Figs. 7 and 8 show a large electric-field-bending effect requires a large value of the fine-tuning factor to make the nonlinearity as low as possible. The fine-tuning factor is more effective for the large electric-field-bending effect.

Fig. 9 shows that the optimum window width of the screen electrode is slightly larger than $3 \varphi_{\mathrm{s}}$, which amounts to about $52^{\circ}$.

\section{EXPERIMENTAL RESULTS}

An angular-position sensor has been built and tested with the structure described in Fig. 1 and the geometrical parameters given in Table I. The common electrode and segmented electrode were made using simple printed-circuitboard technology. Guard electrodes, surrounding the segmented electrode and the common electrode, were used to reduce the influence of the electric-field bending in radial directions and of electromagnetic interference. The rotating screen electrode is grounded by using a sliding contact. A window width of $48^{\circ}$ on the screen electrode was selected by taking account of the influences of the electric-field-bending effect and the mechanical errors. The performance of the sensor was tested using a signal-processing circuit which has a resolution of 2.0 ", an angular test table which has a resolution of 5.0", and a microcontroller. Fig. 10(a) and (b) show the measured nonlinearities of the sensor over a range of one segment width using a fine-tuning factor of $\alpha=0$ and $\alpha=0.0314$, respectively. These nonlinearities are mainly caused by the influence of the electric-field-bending effect. Over a measurement range of $90^{\circ}$, the nonlinearity of the sensor is about \pm 50 " due to the influences of the electric-fieldbending effect, the mechanical errors, such as, nonflatness of electrode, and other nonidealities.

These measured results are in very good agreement with the analytical results shown in Fig. 6. A comparison between Fig. 10(a) and (b) shows that the fine tuning is very effective to reduce the influence of the electric-field-bending effect and the improvement amounts to a factor of 5.8.

\section{CONCLUSION}

Analytical expressions for the distributions of the potential and charge density in a capacitive angular-position sensor with three electrode disks in an axis-symmetrical configuration have been found by using an appropriate physical model and solving Laplace's equation.

These analytical expressions are very useful to calculate the influence of electric-field bending on the nonlinearity in a capacitive angular- and linear-position sensor. It has been shown that this nonlinearity is significantly reduced by using an additional parameter in the retrieving algorithm, the "finetuning factor." Optimal values for this fine-tuning factor have been calculated for a variety of geometrical parameters. The results of the analysis are very useful to find the optimal geometrical parameters with respect to the accuracy of the sensor structure. This optimum will depend on the nonlinearity on the one hand and on the nonflatness, mechanical stability, and sensitivity to pollution and humidity on the other hand. The 


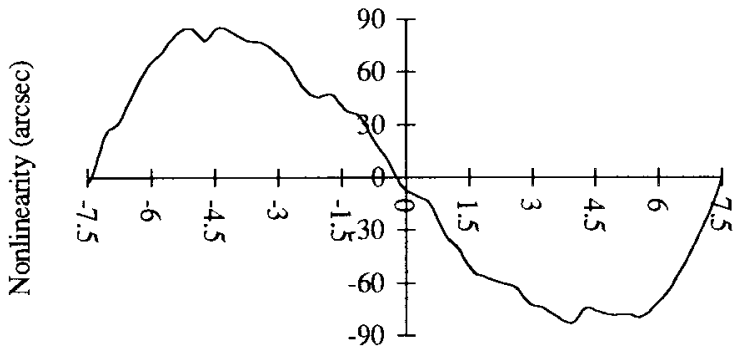

The measured angular position (degree)

(a)

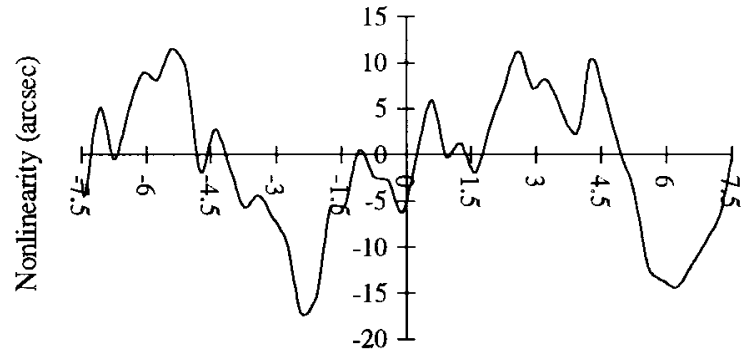

The measured angular position (degree)

(b)

Fig. 10. The measured non-linearity of the sensor (a) for $\alpha=0$, (b) for $\alpha=0.0314$.

physical model has been tested and verified experimentally with an angular-position sensor.

\section{ACKNOWLEDGMENT}

The authors would like to thank J. W. Spronck, Department of Mechanical Engineering and Marine Technology, Delft Univ. of Technology, and M. van der Lee and D. A. J. M. Bertels, Enraf Delft Instruments, for their helpful discussions.

\section{REFERENCES}

[1] F. Zhu, J. W. Spronck, and H. F. van Beek, "A capacitive absolute position transducer," in Proc. 7th Int. Precision Engineering Seminar, Kobe, Japan, 1993.

[2] F. N. Toth and G. C. M. Meijer, "A low-cost, smart capacitive position sensor," IEEE Trans. Instrum. Meas., vol. 41, no. 6, pp. 1041-1044, Dec. 1992.

[3] G. W. de Jong, "Smart capacitive sensor (physical, geometrical and electronic aspects)," Ph.D. dissertation, Delft Univ. of Technology, Faculty of Electrical Engineering, April 1994.

[4] W. C. Heerens, "Review article: Application of capacitance techniques in sensor design," J. Phys. E: Sci. Instrum., vol. 19, pp. 897-906, 1986.

[5] _ , "Multi-terminal capacitor sensors," J. Phys. E: Sci. Instrum., vol. 15 , pp. $137-141,1982$.
[6] M. H. W. Bonse, "Capacitive position transducers, theoretical aspects and practical applications," Ph.D dissertation, Delft Univ. of Technology, Faculty of Mechanical Engineering and Marine Technology, Dec. 1995.

[7] X. J. Li, G. C. M. Meijer and G. W. de Jong, "An accurate smart capacitive angular-position sensor with a full circle range," in Proc. IMTC/95, Watham, MA, April 24-26, 1995, pp. 80-83.

[8] Ansoft Corp., "Maxwell 2-D field simulator," Version 4.3x Release Notes, Pittsburgh, PA, June 1991.

Xiujun Li, photograph and biography not available at the time of publication.

Gerben de Jong, photograph and biography not available at the time of publication.

Gerard C. M. Meijer (M'94), photograph and biography not available at the time of publication. 